\title{
INVESTIGACIONES
}

\section{Validez de constructo de un instrumento para evaluar la promoción de la metacognición en el aula}

\author{
Validity of Instrument Construction to Evaluate the Promotion \\ of Metacognition in the Classroom
}

\author{
Claudia Romo-Sabugal ${ }^{a}$, Luis Gibran Juárez-Hernández ${ }^{b}$, Sergio Tobón ${ }^{b}$ \\ ${ }^{a}$ Universidad Mondragón, México. \\ claudiaromo@live.com.mx \\ ${ }^{b}$ Investigador del Centro Universitario CIFE, México. \\ luisgibran@cife.edu.mx, stobon@cife.edu.mx
}

\begin{abstract}
Se realizó un análisis de validez de constructo y confiabilidad del instrumento "Escala de Intervención Docente Orientada al Desarrollo de la Metacognición en el Aula". La escala integra 29 ítems organizados en seis aspectos: sujeto, tarea, contexto, planeación, monitoreo y evaluación; todos estos aspectos conforman las habilidades metacognitivas que deberán desarrollar los estudiantes. El instrumento se aplicó a 346 docentes de distintos niveles educativos (preescolar, primaria, secundaria, preparatoria y universidad) y el método de estudio fue mediante el análisis factorial exploratorio, verificando previamente la pertinencia de los datos para su abordaje según el método elegido. Los resultados mostraron que un solo factor explicó más de $55 \%$ de la varianza y que en éste se encuentran representados los 22 ítems enunciados, correspondiéndose con lo propuesto teóricamente. La escala muestra un valor óptimo de confiabilidad (Alfa de Cronbach: 0.88). Se concluye que el instrumento representa de forma adecuada las dimensiones del constructo.
\end{abstract}

Palabras clave: confiabilidad, constructo, docentes, evaluación, instrumento y metacognición.

\begin{abstract}
An analysis on the construct validity and reliability on the instrument "Teaching Intervention Scale Towards Metacognition Development in the Classroom" were performed. The scale integrates 29 items organized in six aspects: the individual, the homework, the context, the planning, the monitoring and the evaluation which refer to the metacognitive skills that students should develop. The instrument was applied to 346 teachers of different educational levels (kindergarten, elementary, middle school, high school and college), and the analysis method was through the exploratory factor analysis, previously verifying data pertinence for its analysis through the method being chosen. Results showed that a single factor explained more than $55 \%$ of the variance and that in this factor, 22 offered items were represented, corresponding with what was theoretically proposed. The scale shows an optimal reliability value (Alfa de Cronbach: 0.88). It has been concluded that the instrument represents, very properly, the dimensions of the construct.
\end{abstract}

Key words: reliability, construct, teachers, evaluation, instrument and metacognition. 


\section{INTRODUCCIÓN}

Flavell (1979) definió a la metacognición como el conocimiento de los propios procesos cognitivos, de los resultados de estos procesos y de cualquier aspecto que se relacionara con ellos. Otros autores señalan que la metacognición "es el conocimiento y regulación que poseemos de todos estos procesos básicos, superiores y complejos: qué son, cómo operan, cuándo hay que utilizar uno u otro, qué factores coadyuvan o entorpecen su funcionamiento" (Alama, 2015, p. 78), o bien, una introspección reflexiva, una habilidad que implica la autoobservación, el control ejecutivo, una reflexión sobre las propias acciones y como un proceso de internalización y regulación de los procesos (Báez-Alcaíno y Onrubia-Goñi, 2016). En la literatura se suele resumir esta secuencia diciendo que la metacognición requiere saber qué se quiere conseguir (objetivos) y saber cómo lograrlo (Chrobak, 2004). Es precisamente en estos aspectos en que resulta relevante su promoción en las personas, ya que de ello dependerá su capacidad de pensar con mayor profundidad, amplitud y autonomía.

La metacognición se considera como un elemento importante en el enfoque socioformativo propuesto por Tobón (2013), quien lo define como un marco de reflexiónacción educativo que busca generar las condiciones pedagógicas para facilitarla y promover el desarrollo de personas íntegras, integrales y competentes, que sean capaces de afrontar retos y problemas y de actuar de forma eficiente en diversos contextos, no sólo en su desarrollo personal, sino en otros ámbitos de su vida, como en el social o el ecológico, entre otros. Este enfoque no se centra en el aprendizaje como meta, sino que lo trasciende al considerar a la persona como un todo, por lo que deberá atenderse el desarrollo de sus competencias. El mismo autor refiere que éstas se deben entender como actuaciones integrales de las personas frente a las actividades y los problemas con autonomía.

Si se busca un desarrollo de competencias desde este enfoque, el docente deberá mediar para generar un cambio en el aprendizaje, para que éste se oriente al desarrollo del pensamiento complejo que integra, como una de sus habilidades, la metacognición, esencial en la formación de las nuevas generaciones, que requieren habilidades y herramientas para enfrentarse a diversas situaciones, ya que el reconocimiento de los propios procesos de conocimiento se transfiere al control y a la regulación de éstos (Alama-Flores, 2015).

El problema es cómo puede promoverse el desarrollo de la metacognición en el aula, ya que los docentes frecuentemente orientan sus esfuerzos al dominio de contenidos conceptuales y dejan de lado el desarrollo de habilidades tan trascendentes para la vida de los estudiantes, como la metacognición, que no sólo puede ayudarlos en sus procesos académicos, sino como una forma de enfrentarse a la vida. En este sentido, la promoción de la metacognición debe realizarse a través del empleo de estrategias metacognitivas. Chrobak (2004) las identifica como aquellas relacionadas con el conocimiento del conocimiento y con el control ejecutivo. Ambas resultan indispensables para integrar en la formación de los estudiantes opciones para aprender de manera eficaz y ejercer la autorregulación del aprendizaje.

Hernández e Izquierdo (2016) indican que el empleo de estrategias metacognitivas tiene un impacto positivo en el desempeño de los estudiantes y que incrementan su motivación. Asimismo señalan que este tipo de estrategias debe enseñarse de forma explícita, es decir, que el profesor ayude a sus estudiantes a pensar en las estrategias que le resulten más eficaces, a reflexionar en torno a los procesos cognitivos y metacognitivos que ponen en juego al enfrentarse a una tarea, por lo que no sólo enfatiza la conciencia sobre la 
evaluación, la conducción de su propio proceso de aprendizaje, de los factores cognitivos y afectivos que intervienen en el mismo, sino que también se les brinda la oportunidad de captar y procesar nueva información para integrarla a sus esquemas de conocimiento y poder vincularla a sus conocimientos previos. Los mismos autores coinciden en que la intervención docente deberá orientarse a guiar a sus estudiantes a reconocer cómo y cuándo resulta conveniente emplear determinadas estrategias para lograr mayor éxito académico. Por lo anterior, Jaramillo-Naranjo y Simbaña-Gallardo (2014) señalan que cuando los estudiantes aplican estrategias metacognitivas logran procesar información con mayor facilidad, mejorar su autoconocimiento y de esta forma pueden llegar al autocontrol en sus procesos de aprendizaje.

Para los docentes constituye un reto lograr que sus estudiantes desarrollen habilidades metacognitivas para que sean capaces de enfrentar con éxito los desafíos de la actualidad, ya que como señalan Tamayo, Zona y Loaiza (2016) pensar críticamente implica autocorrección, pensar sobre el pensamiento y generar acciones para mejorarlo, lo que remite al desarrollo metacognitivo que promueven o deberían promover los docentes en su trabajo cotidiano en el aula. Los docentes requieren ser críticos respecto de sus intervenciones con la finalidad de promover las habilidades que requieren los alumnos en la compleja realidad que les toca enfrentar; por ello es necesario considerar que la metacognición resulta indispensable para configurar una nueva perspectiva en los procesos de enseñanza y de aprendizaje que se promueven en las escuelas. De esta forma los docentes deben involucrarse y acceder a conocimientos y estrategias que contribuyan a combatir el bajo rendimiento escolar y potenciar en sus alumnos métodos más eficaces para que logren aprender (Chrobak, 2004).

Es importante incluir en los procesos de instrucción y de formación las características de la metacognición referidas por Burón (1993) que resultan esenciales en el desarrollo metacognitivo de los estudiantes, como llegar a conocer los objetivos que se quiere alcanzar con el esfuerzo mental; la posibilidad de elección de las estrategias para conseguir los objetivos planteados; la autoobservación del propio proceso de elaboración de conocimientos, para comprobar si las estrategias elegidas son las adecuadas y la evaluación de los resultados para saber hasta qué punto se han logrado los objetivos.

Al tomar en cuenta las características de la metacognición e implementar en el aula estrategias conducentes a desarrollarlas, los estudiantes contarán con habilidades y procesos metacognitivos útiles en su vida académica, personal y social. Las estrategias se emplean de forma consciente y son orientadas hacia un fin, como señalan Valle-Arias, Barca-Lozano, González-Cabanach y Núñez-Pérez (1999), quienes además identifican las estrategias metacognitivas como aquellas que hacen referencia a la planificación, el control y la evaluación de la propia cognición; permiten el conocimiento de los procesos mentales y la regulación de los mismos y permiten a los estudiantes la posibilidad de transferirlas a otras situaciones, considerando las variables que intervienen en dicho proceso: de la persona (consciencia y el conocimiento que el sujeto tiene de sí mismo, de sus capacidades y limitaciones cognitivas), de la tarea (respecto del problema a resolver, considerando el objetivo, grado de dificultad) y las de estrategia (conocimiento sobre las estrategias aplicables en la situación a enfrentar). Por lo anterior resulta fundamental la evaluación de los docentes en el desarrollo de la metacognición de los alumnos.

Diversos aportes instrumentales se han implementado para identificar logros en el desarrollo metacognitivo de los estudiantes, dichos instrumentos se han orientado a su 
empleo en determinadas áreas del conocimiento, como el Cuestionario de Estrategias Metacognitivas de Comprensión de Textos Narrativos (Larrañaga \& Yubero, 2015), o la Escala de Conciencia Lectora (Jiménez-Rodríguez, Puente-Ferreras, Alvarado-Izquierdo \& Arrebillaga, 2009); o bien el instrumento Reflexiones sobre el Estudio Universitario (Palacios \& Schinella, 2017).

Por su parte, los aportes instrumentales sobre las estrategias empleadas por los profesores para favorecer el aprendizaje de sus alumnos se cuentan con la Escala de Estrategias Docentes para Aprendizajes Significativos (Méndez-Hinojosa \& González Ramírez, 2011), la Escala Metacognitiva (Jaramillo y Osses, 2012) la cual está orientada a medir la metacognición en términos de conocimiento, experiencias metacognitivas y autorregulación cognitiva. Por su parte, Huerta-Bustos, Vega-Bravo y Galindo-León (2014) adaptaron el instrumento "Inventario de Habilidades Metacognitivas" (Metacognitive Awareness Inventory) que fue creado por Schraw \& Denninson (1994) en la Universidad de Nebraska, para identificar habilidades metacognitivas, conocido como MAI, por sus siglas en inglés. Este último está compuesto de ocho categorías (conocimiento declarativo, procedimental, condicional, planificación, organización, monitoreo, depuración y evaluación).

Un aspecto para destacar de los últimos instrumentos mencionados es que, si bien abordan habilidades metacognitivas en los estudiantes, no permiten al docente orientar su intervención hacia este desarrollo por estar dirigidos hacia la autoevaluación de los propios estudiantes; estos instrumentos no están diseñados para que el docente valore su propia actuación en el aula orientada a este desarrollo tan trascendente para la vida de los individuos como lo es la metacognición. A este respecto, los aportes instrumentales que evalúen el grado en el cual el docente promueve la metacognición en sus estudiantes son inexistentes.

Frente a esta necesidad resulta indispensable implementar la enseñanza explícita de estrategias metacognitivas en todos los niveles educativos, lo cual no será posible si los docentes no toman conciencia de sus intervenciones y reflexionan sobre si éstas se orientan a que sus alumnos aprendan a aprender. En este sentido la Escala de Intervención Docente Orientada a la Promoción de la Metacognición en el Aula (EIDOPMA) (RomoSabugal, Tobón \& Juárez-Hernández, 2020.) constituye un instrumento que busca ayudar a los docentes a identificar las estrategias que promueven y aquellas que sería conveniente implementar con la intención de favorecer este desarrollo en sus estudiantes. Este instrumento integra aquellos aspectos que el docente debe implementar para promover el desarrollo metacognitivo de sus estudiantes mediante 29 ítems organizados en seis aspectos: el sujeto, la tarea, el contexto, la planeación, el monitoreo y la evaluación; éstos conforman las habilidades metacognitivas que deberán desarrollar los estudiantes. Es importante precisar que el instrumento fue validado en términos de contenido mediante el juicio de expertos y se verificó su asequibilidad mediante la aplicación a un grupo piloto.

En relación con esto, para un instrumento de medición es importante contar no sólo con la validación de contenido, sino con una propiedad psicométrica denominada validez de constructo, la cual es referida como la principal de los tipos de validez (Pérez-Gil, ChacónMoscoso \& Moreno-Rodríguez, 2000), ya que verifica si la estructura del instrumento reproduce realmente la del constructo planteado (Lagunes, 2017). Por lo anterior el presente estudio tiene como objetivo efectuar un análisis de validez de constructo y confiabilidad del EIDOPMA. 


\section{METODOLOGÍA}

\subsection{TIPO DE ESTUDIO}

Se realizó un estudio instrumental, el cual se destina al desarrollo de pruebas, tanto diseñadas como adaptadas, y al estudio de sus propiedades psicométricas (Montero \& León, 2005). En el presente se analizaron las propiedades psicométricas de validez de constructo y confiabilidad.

\subsection{PROCEDIMIENTO}

El estudio se llevó a cabo mediante las siguientes fases:

\subsubsection{Instrumento}

El instrumento empleado fue la EIDopma (Escala de Intervención Docente Orientada a la Promoción de la Metacognición en el Aula) (Romo-Sabugal et al., 2020). Dicho instrumento se diseñó considerando aquellos aspectos que el docente debe implementar para promover el desarrollo metacognitivo de sus estudiantes, para ello se tomaron en cuenta las aportaciones de Brown (1987), Flavell (1987), Flórez, Mondragón, Pérez \& Torrado (2003), Castañeda-Figueiras (2004), Bruning, Schraw, Norby \& Ronning (2005) y el enfoque socioformativo propuesto por Tobón (2013). Específicamente el instrumento abarca tres preguntas de opción múltiple relacionadas con las creencias docentes en relación con los factores que influyen en el aprendizaje de los estudiantes y una escala de Likert integrada por 29 ítems en torno al factor "Metacognición" tomando en cuenta dos subprocesos metacognitivos, que están íntimamente ligados entre sí: el orientado a la cognición y el orientado a la regulación de la cognición. Como se indicó, posteriormente a su construcción, se validó en contenido y se obtuvo un valor óptimo de confiabilidad, así como un buen nivel de comprensión de ítems e instrucciones en la aplicación a un grupo piloto (Romo-Sabugal et al., 2020).

\subsubsection{Selección de la muestra poblacional para la aplicación del instrumento}

Para el presente se consultó el número total de docentes que laboran en los diferentes niveles educativos en el estado de Querétaro. Posterior a este proceso, se calculó el tamaño de muestra para poblaciones finitas, empleando una herramienta en línea y se estableció como parámetro de entrada un nivel del $95 \%$ de confianza. En el cuadro 1, se integran los resultados de la muestra obtenida. 
Estudios Pedagógicos XLVII Nº 3: 191-205, 2021

VALIDEZ DE CONSTRUCTO DE UN INSTRUMENTO PARA EVALUAR LA PROMOCIÓN DE LA METACOGNICIÓN EN EL AULA

Tabla 1. Muestra de docentes correspondiente a cada nivel educativo en el estado de Querétaro

\begin{tabular}{|l|c|c|}
\hline \multicolumn{1}{|c|}{ Nivel educativo } & Total de docentes & Muestra correspondiente \\
\hline Preescolar & 4.033 & 363.90 \\
\hline Primaria & 8.390 & 381.79 \\
\hline Secundaria & 5.716 & 373.83 \\
\hline Bachillerato & 6.483 & 376.90 \\
\hline Licenciatura & 8.672 & 382.36 \\
\hline \multicolumn{1}{|c|}{ Total } & 33.861 & 395.52 \\
\hline
\end{tabular}

A partir del cálculo de la muestra, se eligieron 395 docentes. Finalmente, la recolección de éstos integró un total de 346 instrumentos respondidos por docentes (cuadro 2), de los cuales 55 fueron de nivel preescolar, 85 de nivel primaria, 55 de nivel secundaria, 51 de nivel bachillerato y 100 de nivel superior.

Tabla 2. Datos sociodemográficos de los participantes

\begin{tabular}{|l|l|}
\hline \multicolumn{1}{|c|}{ Sexo } & \multicolumn{1}{|c|}{$\begin{array}{c}\text { Hombres 30\% } \\
\text { Mujeres 70\% }\end{array}$} \\
\hline Promedio de edad & 48 años \\
\hline Promedio de años de estudio & 23 años \\
\hline Condiciones económicas & $\begin{array}{l}\text { Alta 18\% } \\
\text { Media 70\% } \\
\text { Baja 12\% }\end{array}$ \\
\hline Zona de residencia & $\begin{array}{l}\text { Ciudad de Querétaro 65\% } \\
\text { Zonas aledañas 35\% }\end{array}$ \\
\hline Situación laboral & Docentes frente a grupo \\
\hline
\end{tabular}

\subsection{ANÁLISIS DE VALIDEZ DE CONSTRUCTO Y CONFIABILIDAD}

La validez de constructo es un concepto que integra la validez de contenido y de criterio en un marco común referido a relaciones teóricamente relevantes (Pérez-Gil, ChacónMoscoso \& Moreno-Rodríguez, 2000). Específicamente en el presente, el análisis de validez de constructo se efectuó mediante la técnica de análisis factorial exploratorio. De acuerdo con el tamaño de muestra (346) se cumplió con el supuesto de la muestra mínima para efectuar esta técnica estadística (Costello \& Osborne, 2005; Mavrou, 2015). Para determinar la pertinencia de los datos para este análisis se realizó mediante el cálculo de la determinante de la matriz de correlaciones, la prueba de Kaiser Meyer Olkin (KMO>0.70) 
y de Barlett ( $\mathrm{p}<0.05)$ (Gorsuch, 1983; Costello \& Osborne, 2005; Mavrou, 2015; MéndezMartínez \& Rondón-Sepúlveda, 2012; Juárez-Hernández; 2018). Una vez verificados estos supuestos, se seleccionó el método de extracción de factorización de ejes principales debido a que es robusto ante el supuesto de violación de normalidad y correlaciones moderadas (Winter \& Doudou, 2012). El número de factores a retener se basó en la regla de GutmanKaiser y la significancia de las cargas factoriales se fundamentó en la propuesta de RositasMartínez (2014). Si se presentase un ítem con carga factorial significativa a más de un factor, se realizaría la rotación de la matriz mediante el algoritmo de mayor conveniencia. Finalmente, el análisis de confiabilidad se realizó mediante el coeficiente Alfa de Cronbach (Cronbach, 1951), teniendo en cuenta los valores establecidos por Nunally y Bernstein (1994) para el valor del coeficiente.

\section{CONSIDERACIONES ÉTICAS}

En el cuestionario entregado a cada participante se integró el compromiso de mantener su privacidad y emplear los datos únicamente para lo que fue diseñado el instrumento, lo que constituye un ejercicio ético y responsable en el tratamiento de datos estadísticos.

\section{RESULTADOS}

La determinante de la matriz de correlaciones (d: 0.0000000390), la prueba de adecuación (KMO: 0.963) y la esfericidad de Bartlett $\left(\mathrm{X}^{2}: 6513.383\right.$, gl: 406, $\left.\mathrm{p}<0.05\right)$ denotaron la pertinencia de los datos para ser analizados mediante el AFE. Este análisis mostró la adecuada representación de los ítems dentro del modelo factorial (i.e., comunalidades). En este sentido, se precisa que un solo factor presentó autovalor superior a uno, y éste explicó más del $50 \%$ de la varianza. La matriz factorial reveló que todos los ítems presentan una alta carga hacia el factor uno (cuadro 3). Finalmente se refiere que, de la muestra de 346 participantes, se determinó que el instrumento posee una alta consistencia interna (Alfa de Cronbach: 0.88). 
Tabla 3. Matriz factorial

\begin{tabular}{|c|c|c|c|}
\hline Aspecto & Ítem & Comunalidad & Carga factorial \\
\hline El sujeto & $\begin{array}{l}\text { 1. Sensibiliza a sus alumnos hacia el estudio, a } \\
\text { tener como estudiantes actitudes positivas, a poner } \\
\text { atención y a concentrarse. }\end{array}$ & 0.548 & 0.729 \\
\hline El sujeto & $\begin{array}{l}\text { 2. Ayuda a sus alumnos a reconocer sus estados } \\
\text { emocionales, su atención y su concentración } \\
\text { mientras trabajan. }\end{array}$ & 0.721 & 0.717 \\
\hline El sujeto & $\begin{array}{l}\text { 3. Orienta a sus alumnos sobre cómo poner } \\
\text { atención, concentrarse y evitar distraerse. }\end{array}$ & 0.619 & 0.694 \\
\hline El sujeto & $\begin{array}{l}4 \text { Ayuda a sus alumnos a identificar problemas y } \\
\text { dificultades que experimentan en su proceso de } \\
\text { aprendizaje. }\end{array}$ & 0.56 & 0.709 \\
\hline El sujeto & $\begin{array}{l}\text { 5. Promueve en sus alumnos el autoconocimiento y } \\
\text { el deseo de superarse a sí mismos, de ser } \\
\text { congruentes y vivir conforme a sus valores con un } \\
\text { proyecto ético de vida. }\end{array}$ & 0.547 & 0.73 \\
\hline La tarea & $\begin{array}{l}\text { 6. Brinda a sus alumnos la posibilidad de practicar } \\
\text { ciertas acciones y tareas para asegurar que logren } \\
\text { realizarlas con éxito. }\end{array}$ & 0.486 & 0.681 \\
\hline La tarea & $\begin{array}{l}\text { 7. Favorece el intercambio de ideas en torno a la } \\
\text { realización de actividades colectivas, a problemas } \\
\text { del contexto y a la colaboración y sobre su impacto } \\
\text { en la realidad. }\end{array}$ & 0.475 & 0.642 \\
\hline La tarea & 8. Les enseña técnicas para aprender mejor. & 0.525 & 0.709 \\
\hline La tarea & $\begin{array}{l}\text { 9. Enseña a sus alumnos a emplear y manejar } \\
\text { herramientas de aprendizaje y materiales que } \\
\text { faciliten resolver situaciones y lograr aprendizajes } \\
\text { posteriores. }\end{array}$ & 0.582 & 0.738 \\
\hline La tarea & $\begin{array}{l}\text { 10. Emplea diversas estrategias de trabajo para que } \\
\text { los alumnos construyan su aprendizaje. }\end{array}$ & 0.583 & 0.716 \\
\hline El contexto & $\begin{array}{l}\text { 11. Plantea situaciones retadoras que los lleven a } \\
\text { buscar soluciones. }\end{array}$ & 0.507 & 0.698 \\
\hline El contexto & $\begin{array}{l}\text { 12. Cuestiona sobre la conveniencia emplear } \\
\text { ciertas formas de realizar una tarea. }\end{array}$ & 0.436 & 0.653 \\
\hline El contexto & $\begin{array}{l}\text { 13. Pregunta a sus alumnos: ¿para qué te puede } \\
\text { servir? ¿De qué otra forma podrías...? Favorece la } \\
\text { transferencia de lo aprendido a otras situaciones. }\end{array}$ & 0.525 & 0.717 \\
\hline El contexto & $\begin{array}{l}\text { 14. Promueve la iniciativa y la creatividad en sus } \\
\text { alumnos para resolver situaciones e influir en } \\
\text { cambios de su realidad inmediata. }\end{array}$ & 0.52 & 0.711 \\
\hline El contexto & $\begin{array}{l}\text { 15. Vincula los conocimientos adquiridos con otras } \\
\text { áreas del saber, ejemplifica y aplica lo aprendido } \\
\text { en otras situaciones. Promueve la transferencia de } \\
\text { lo aprendido a otras situaciones. }\end{array}$ & 0.537 & 0.703 \\
\hline
\end{tabular}




\begin{tabular}{|c|c|c|c|}
\hline Planeación & $\begin{array}{l}\text { 16. Plantea a sus alumnos metas y objetivos a } \\
\text { lograr con las actividades a realizar. }\end{array}$ & 0.5 & 0.706 \\
\hline Planeación & $\begin{array}{l}\text { 17. Implementa el uso de agenda, calendario y } \\
\text { otros apoyos para ayudarlos a organizar sus } \\
\text { trabajos y deberes. }\end{array}$ & 0.388 & 0.558 \\
\hline Planeación & $\begin{array}{l}\text { 18. Establece reglas para realizar ciertas tareas y } \\
\text { participar en las actividades. }\end{array}$ & 0.567 & 0.688 \\
\hline Planeación & $\begin{array}{l}\text { 19. Sus alumnos participan en la planeación del } \\
\text { trabajo a realizar aportando ideas. Aprovecha las } \\
\text { sugerencias de sus alumnos sobre el trabajo de } \\
\text { clase. }\end{array}$ & 0.401 & 0.592 \\
\hline Monitoreo & $\begin{array}{l}\text { 20. Ayuda a sus alumnos a identificar y superar } \\
\text { dificultades que se van presentando en la } \\
\text { realización de sus trabajos implementando } \\
\text { acciones que permitan ir mejorando en el proceso } \\
\text { de aprendizaje. }\end{array}$ & 0.544 & 0.725 \\
\hline Monitoreo & $\begin{array}{l}\text { 21. Ayuda a sus alumnos a reconstruir el itinerario } \\
\text { seguido en la resolución de un problema o en la } \\
\text { realización de alguna tarea o actividad. }\end{array}$ & 0.624 & 0.737 \\
\hline Monitoreo & $\begin{array}{l}\text { 22. Conduce la realización de las actividades } \\
\text { recordando los objetivos, cómo deben realizarlas y } \\
\text { qué deben tomar en cuenta para ello. }\end{array}$ & 0.573 & 0.743 \\
\hline Monitoreo & $\begin{array}{l}\text { 23. Guía la reflexión de sus alumnos sobre cómo } \\
\text { van abordando las tareas y actividades con } \\
\text { preguntas: ¿Cómo vas? ¿Vas bien? ¿Qué te falta? } \\
\text { ¿Qué puedes hacer para...? }\end{array}$ & 0.51 & 0.711 \\
\hline Evaluación & $\begin{array}{l}\text { 24. Guía la reflexión en sus alumnos sobre qué } \\
\text { aprendieron, cómo y para qué. }\end{array}$ & 0.514 & 0.715 \\
\hline Evaluación & $\begin{array}{l}\text { 25. Retroalimenta el trabajo realizado por sus } \\
\text { alumnos. }\end{array}$ & 0.541 & 0.713 \\
\hline Evaluación & $\begin{array}{l}\text { 26. Ayuda a sus alumnos a reconocer sus logros, } \\
\text { talentos y las dificultades experimentadas en su } \\
\text { proceso de aprendizaje. }\end{array}$ & 0.62 & 0.787 \\
\hline Evaluación & $\begin{array}{l}\text { 27. Facilita la autoevaluación en sus alumnos } \\
\text { empleando algunos instrumentos. }\end{array}$ & 0.519 & 0.676 \\
\hline Evaluación & $\begin{array}{l}\text { 28. Promueve la revisión de los alumnos en sus } \\
\text { trabajos y productos terminados. }\end{array}$ & 0.467 & 0.646 \\
\hline Evaluación & $\begin{array}{l}\text { 29. Promueve la revisión y la reconstrucción del } \\
\text { itinerario seguido en la realización de una } \\
\text { actividad. }\end{array}$ & 0.687 & 0.703 \\
\hline
\end{tabular}




\section{DISCUSIÓN}

La metacognición es entendida como el conocimiento de los propios procesos mentales y hace referencia a la supervisión activa y a la regulación consecuente de los mismos, así como a su organización (Flavell, 1979). Esta definición fue retomada por Carretero (2001) al identificarla como el conocimiento que las personas construyen en relación con su funcionamiento cognitivo vinculado a la supervisión y la regulación de la propia actividad cognitiva, lo cual se concreta en el conocimiento y el control metacognitivo, que a su vez involucra al conocimiento de la propia persona, de la tarea a realizar y de la estrategia (BáezAlcaíno \& Onrubia Goñi, 2016). En este sentido la intervención docente no puede omitir intervenciones orientadas al desarrollo metacognitivo que favorezcan en sus estudiantes el control de sus procesos de aprendizaje, ayudándolos a convertirse en aprendices competentes, participantes activos, capaces de conducir y autorregular su propio aprendizaje. Dichas intervenciones deberán considerar cuatro tipos de estrategias metacognitivas: planificación, supervisión, regulación y evaluación (Báez-Alcaíno \& Onrubia-Goñi, 2016).

Por ello resulta indispensable que los docentes tengan presente que el conocimiento de estas estrategias por parte de sus estudiantes está íntimamente ligado a la motivación, el autoconcepto y la autoestima de cada sujeto como aprendiz e influyen y dirigen su proceder, no sólo en actividades académicas, sino en las esperanzas, las actitudes y los niveles de aspiración en la vida. Por ello, al considerar estos aspectos esenciales que influyen en el aprendizaje deberán privilegiar, como lo refiere Ospina-Rodríguez (2006), la motivación intrínseca que procede precisamente del propio sujeto y cuyo objetivo es experimentar la autorrealización, la satisfacción por el logro de una meta, los estudiantes encuentran el incentivo en la propia tarea.

En este sentido, los aportes instrumentales reportados únicamente se han enfocado en efectuar una medición de las estrategias que emplean los docentes para promover el aprendizaje significativo (Méndez-Hinojosa \& González Ramírez, 2011), pero no específicamente a la promoción de la metacognición en el trabajo del aula; como es el caso de la Escala de Estrategias Docentes para Aprendizajes Significativos (ELEDAS) (MéndezHinojosa \& González Ramírez, 2011). Sin embargo, aunque algunas de sus estrategias implican el empleo de habilidades metacognitivas, no está diseñada para que el docente valore su propia intervención en el aula orientada a este desarrollo tan trascendente para la vida de los individuos como lo es la metacognición. Por ello resulta necesario contar con una herramienta mediante la cual sea el mismo docente quien se autoevalúe y reconozca qué acciones realiza y qué aspectos requiere implementar para asegurar el desarrollo metacognitivo de sus estudiantes a través de su intervención en el trabajo escolar.

Por lo anterior es fundamental contar con un instrumento que aborde este aspecto desde la perspectiva de los docentes, por lo que el diseño del instrumento aquí presentado constituye un avance, ya que incorpora en sus ítems al sujeto como aprendiz activo tomando en cuenta el aspecto cognitivo y emocional; la tarea considerando las acciones requeridas para alcanzar una meta; el contexto, que brinda información situacional para tomar decisiones; la planeación para orientar sus actuaciones; el monitoreo para redirigir sus esfuerzos, y la evaluación para valorar sus propios resultados. En este sentido, el instrumento EIDOPMA viene a fungir esa función considerando que es un instrumento útil para ayudar a los docentes a identificar las estrategias que emplean y/o dejan de emplear en el aula orientadas al desarrollo metacognitivo de sus estudiantes, ya que al aplicarla podrán 
reflexionar sobre la forma en que realizan su intervención en el aula y si verdaderamente promueven la metacognición, clave para el aprendizaje permanente. Asimismo, la herramienta les permitirá reconocer cuáles son las estrategias menos empleadas para implementarlas y de esta forma favorecer el desarrollo de habilidades metacognitivas en el aula. En relación con esto, otro aspecto que se incluye dentro del instrumento es el enfoque socioformativo, el cual considera la metacognición como un aspecto necesario para que el sujeto logre conducirse como una persona íntegra y competente al actuar de manera eficiente en todos los ámbitos de su vida y de esta forma contribuir a transformar su realidad (Tobón, 2013).

Un aspecto relevante del instrumento EIDOPMA es que, si bien en sus fases iniciales fue sometido a un proceso de revisión y evaluación por expertos, denotaría que los elementos implícitos están relacionados con el constructo metacognición y se requiere del análisis de validez de constructo. En este sentido, al considerar la palabra constructo se hace referencia a un concepto inobservable, por lo que resulta necesario analizar si los elementos son pertinentes y cuantificables para el constructo abordado (Carretero-Dios \& Pérez, 2005). De lo anterior surge la importancia del término validez de constructo, el cual se indica como el concepto unificador que integra las consideraciones de validez de contenido y de criterio en un marco común para probar hipótesis acerca de relaciones teóricamente relevantes (Messick, 1980). En el presente, este análisis se realizó mediante la técnica de análisis factorial exploratorio que, de acuerdo con Lloret-Segura, FerreresTraver, Hernández-Baeza y Tomás-Marco (2014), es una técnica que permite explorar un conjunto de variables o factores que explican las respuestas a los ítems de un test. Es decir, se aplica con la finalidad de comprobar hasta qué punto un instrumento y los ítems que lo integran representan de forma adecuada las dimensiones del constructo (Mavrou, 2015).

Propiamente, los resultados de este análisis revelaron la representación de todos los ítems al modelo factorial (comunalidades y cargas factoriales) y se manifiesta la concordancia del modelo propuesto teóricamente ya que únicamente se refiere un solo factor, la metacognición, y en éste se encuentran representados todos los ítems. De acuerdo con lo anterior, en la propuesta teórica se precisan dos fases de procesos metacognitivos, el orientado a la cognición y el orientado a la regulación de la cognición. Si bien se refieren como dos fases, en el modelo teórico-propuesto estás constituyen una misma dimensión. Con base en esto, ambas fases están articuladas bajo el mismo constructo, como lo señaló Flavell (1979), al explicar que la metacognición implica el conocimiento y el control que el propio individuo tiene de sus procesos cognitivos, los cuales facilitan el aprendizaje e intervienen en la realización de diversas tareas. Es así como existe una relación de intercambio entre ambos (Campo, Escorcia, Moreno \& Palacio, 2016). Burón (1993), al considerar las aportaciones de Flavell y de Brown, sugirió denominar conocimiento autorreflexivo o bien, intracognición para referir más claramente el término metacognición e identificó dos aspectos implicados en este constructo: el relacionado con el conocimiento de la cognición que integra el conocimiento del objetivo, la elección de las estrategias más adecuadas para alcanzarlo, la autoobservación de la ejecución y la evaluación; el otro aspecto es el relacionado con la regulación (control) de la actividad mental que exige planificar antes de enfrentar una tarea, observar la eficacia de la actividad (monitorear) y finalmente comprobar los resultados. Ambos aspectos están contemplados en el constructo metacognición que se buscó medir con la EIDOPMA. Respecto de la confiabilidad se precisa que es alta ya que se obtuvo un resultado óptimo (Alfa de Cronbach: 0.88), lo cual refleja 
que la aplicación del EIDOPMA produce resultados consistentes y coherentes, y refleja la correlación entre ítems y representación del concepto abordado (Welch \& Comer, 1989; Oviedo \& Campo-Arias, 2005).

El proceso metodológico efectuado en el presente tuvo la premisa de brindar un instrumento de evaluación con un carácter científico mediante el cual se asegurará la obtención de evidencias válidas y confiables (Kerlinger \& Lee, 2002; Mendoza-Mendoza \& Garza, 2009). Por lo tanto, el instrumento EIDOPMA tendrá utilidad para los docentes ya que éstos podrán generar un diagnóstico sobre cuáles serían sus áreas de oportunidad en relación con la promoción del desarrollo metacognitivo de sus estudiantes. El detectar las áreas de oportunidad permitirá clarificar las estrategias que se deben promover y emplear en el trabajo escolar para contribuir al desarrollo de éste y que pueda orientar a los docentes respecto de lo que realizan o deben realizar con esta finalidad.

La metacognición está vinculada con la calidad de la educación al estar orientada al aprendizaje estratégico que permitirá a los estudiantes desarrollar habilidades que les aseguren aprender a aprender a lo largo de su vida. Son los docentes quienes tienen en sus manos, a través de su interacción con los alumnos y de su intervención en el trabajo del aula, la oportunidad de promover el desarrollo de habilidades metacognitivas al emplear la EIDOPMA, para identificar las estrategias que promueven o que dejan de promover en sus alumnos y que en la actualidad constituyen un desarrollo importante para favorecer actuaciones conscientes tanto en sus actividades académicas como en su vida personal. Además, es un instrumento aplicable a los diferentes niveles educativos, y de esta forma ayuda al docente a identificar las estrategias que debe implementar para asegurar un desarrollo metacognitivo en sus estudiantes que les permita seguir aprendiendo. AlamaFlores (2015) señala que es el docente quien tiene la responsabilidad y está involucrado de forma directa con el desarrollo metacognitivo de sus estudiantes, es quien debe potenciar este desarrollo en sus estudiantes, que a su vez les permitirá acceder a otros aprendizajes, y retomando las aportaciones de Monereo, enfatiza que es el docente quien debe ayudar a sus alumnos a lograr un autoconocimiento como aprendices, a reflexionar sobre la forma en que aprenden y ayudarlos a tomar decisiones que les permitan regular y monitorear sus acciones, al planear, ejecutar y valorar sus propios procesos de aprendizaje.

Una de las necesidades que se deriva del presente estudio es diseñar un instrumento que permita a los estudiantes identificar y monitorear las estrategias metacognitivas que emplean al desempeñarse como tales, dicho instrumento deberá adecuarse a cada nivel educativo para que pueda ser comprendido y empleado como una herramienta para aprender a aprender. El docente deberá intervenir con la finalidad de hacer explícito para los alumnos el empleo de las estrategias metacognitivas (Alama-Flores, 2015) para que sean capaces de transferirlas a nuevas situaciones y emplearlas, lo que será posible con un entrenamiento en la aplicación de estas.

\section{REFERENCIAS BIBLIOGRÁFICAS}

Alama-Flores, C. M. (2015). Hacia una didáctica de la metacognición. Horizonte de la Ciencia, 5(8), 77-86. Recuperado desde: goo.gl/13b79o

Báez-Alcaíno, J. \& Onrubia-Goñi, J. (2016). Una revisión de tres modelos para enseñar habilidades del pensamiento. Perspectiva Educacional, Formación de Profesores. Pontificia Universidad Católica de Valparíso, 55(1), 94-113. Recuperado desde: goo.gl/gXgBww 
Brown, A. L. (1987). Metacognition, executive control, self-regulation, and other more mysterious mechanisms. In F. E. Weinert \& R. H. Kluwe (Eds.), Metacognition, motivation, and understanding (pp. 65-116). Hillsdale, New Jersey: Lawrence Erlbaum Associates.

Burón, O. P. (1993). Enseñar a aprender: Introducción a la metacognición. Bilbao: Mensajero.

Brunning, R., Schraw, G. \& Norby, M. (2011). Psicología cognitiva y de la instrucción. Madrid: Pearson Educación.

Campo, K., Escorcia, D., Moreno, M. \& Palacio, J. (2016). Metacognición, escritura y rendimiento académico en universitarios de Colombia y Francia. Avances en Psicología Latinoamericana, 34(2), 233-252. doi: http://dx.doi.org/10.12804/apl34.2.2016.03

Carretero, M. (2001). Metacognición y educación. Buenos Aires: Aique.

Carretero-Dios, H. \& Pérez, C. (2005). Normas para el desarrollo y revisión de estudios instrumentales. International Journal of Clinical and Health Psicology, 5(3), 521-551. Recuperado desde: goo. $\mathrm{gl} / \mathrm{cK} 8 \mathrm{ZNo}$

Castañeda-Figueiras, S. (2004). Evaluando y fomentando el desarrollo cognitivo y el aprendizaje complejo. Psicología desde el Caribe, 13, 109-143. Recuperado desde: goo.gl/QXjkyN

Chrobak, R. (2004). La metacognición y las herramientas didácticas. Investigación Didáctica. Universidad Nacional del Comahue, 22(1). Recuperado desde: goo.gl/33xYmY

Costello, A. B. \& Osborne, J. W. (2005). Best practices in exploratory factor analysis: four recommendations for getting the most from your analysis. Practical Assessment, Research \& Evaluation, 10(7), 1-9. Recuperado desde: goo.gl/qbo48j

Cronback, L. J. (1951). Coefficient Alpha and internal structure of test. Psicometrika, 16(3), 1-38. Recuperado desde: goo.gl/L8mjoA

Escobar-Pérez, J. \& Cuervo-Martínez, A. (2008). Validez de contenido y juicio de expertos: una aproximación a su utilización. Avances en Medición, 6, 27-38. Recuperado desde: goo.gl/SKexoE

Flavell, J. H. (1979). Metacognition and cognitive monitoring: a new area of cognitive-developmental inquiry. American Psychologist, 34(10), 906-911. Recuperado desde: goo.gl/pSRTV7

(1987). Speculations about the nature and development of metacognition. In F. E. Weinert \& R. H. Kluwe (Eds.), Metacognition, Motivation and Understanding (pp. 21-29). Hillside, New Jersey: Lawrence Erlbaum Associates.

Flórez, R., Mondragón, S. P., Pérez, C. \& Torrado, M.C. (2003). Explorando la metacognición: evidencia en actividades de lectura y escritura en niños y niñas de 5 a 10 años de edad. Revista Colombiana de Psicología, 12, 85-98. Recuperado desde: goo.gl/a8niHB

Gorsuch, R. L. (1983). Factor analysis (2nd Ed.). Hillsdale, NJ: Lawrence Erlbaum.

Hernández, J. \& Izquierdo, J. (2016). Metacognición y comprensión oral: observación de la práctica docente en nivel universitario. Revista Electrónica de Investigación Educativa, 18(1), 39-52. Recuperado desde: goo.gl/66fE51

Huerta-Bustos, A. P., Vega-Bravo, G. J. \& Galindo-León, M. (2014). Validación del instrumento "Inventario de Habilidades Metacognitivas" (MAI) con estudiantes colombianos. Praxis \& Saber. Revista de Investigación Pedagógica. Maestría en Educación. UPTC, 5(10), 55-74. Recuperado desde: goo.gl/8i8XL5

Jaramillo-Naranjo, L. M. \& Simbaña-Gallardo, V. P. (2014). La metacognición y su aplicación en herramientas virtuales desde la práctica docente. Sophia, Colección de Filosofía de la Educación, 16, 299-313. Recuperado desde: goo.gl/aApCVG

Jaramillo, S. \& Oses, S. (2012). Validación de un Instrumento sobre Metacognición para Estudiantes de Segundo Ciclo de Educación General Básica. Estudios Pedagógicos, 38(2), 117-131. Recuperado desde: goo.gl/RRQ1cp

Jiménez-Rodríguez, V., Puente-Ferreras, A., Alvarado-Izquierdo, J. M. \& Arrebillaga-Durante, L. (2009). Medición de estrategias metacognitivas mediante la Escala de Conciencia Lectora: ESCOLA. Electronic Journal of Research in Educational Psychology, 7(2), 779-804. Recuperado desde: goo.gl/FVFWPd 
Juárez-Hernández, L. G. (2018). Manual práctico de estadística básica para la investigación. Mount Dora, Florida: Kresearch.

Kerlinger, F. \& Lee, H. (2002). Investigación del comportamiento. Métodos de investigación en ciencias sociales. México, D. F.: MacGraw Hill.

Lagunes, C. R. (2017). Recomendaciones sobre procedimientos de construcción y validación de instrumentos y escalas de medición en psicología de la salud. Psicología y Salud, 27(1), 5-18.

Larrañaga, E. \& Yubero, S. (2015). Evaluación de las estrategias metacognitivas de comprensión de textos narrativos Ocnos. Revista de Estudios sobre Lectura, 14, 18-27. Recuperado desde: goo. $\mathrm{gl} / \mathrm{CDJUHt}$

Lloret-Segura, S., Ferreres-Traver, A., Hernández-Baeza, A. \& Tomás-Marco, I. (2014). El análisis factorial exploratorio de los ítems: una guía práctica, revisada y actualizada. Anales de Psicología, 30(3), 1151-1169. Recuperado desde: goo.g1/PoqhXv

Mavrou, I. (2015). Análisis factorial exploratorio: cuestiones conceptuales y metodológicas. Revista Nebrija de Lingüística Aplicada a la Enseñanza de las Lenguas, 19. Recuperado desde: goo.gl/ y6p39b

Méndez-Hinojosa, L. M. \& González-Ramírez, M. T. (2011). Escala de Estrategias Docentes para Aprendizaje Significativo: diseño y evaluación de sus propiedades psicométricas. Revista Electrónica Actualidades Investigativas, 11(3), 1-39. Recuperado desde: goo.gl/YCCuxA

Méndez-Martínez, C. \& Rondón-Sepúlveda, M. (2012). Introducción al análisis factorial exploratorio. Revista Colombiana de Psiquiatría, 41(1), 197-207.

Mendoza-Mendoza, J. \& Garza, J. B. (2009). La medición en el proceso de investigación científica. Evaluación de validez de contenido y confiabilidad. Innovaciones de Negocios, 6(11), 17-32. Recuperado desde: https://goo.gl/qZKnb5

Messick, S. (1980). Test validity and ethics of assessment. American Psychologist, 35(11), 1012-1027. (1998). Test validity: a matter of consequence. Social Indicators Research, 45, 35-44. Recuperado desde: goo.gl/iig2fN

Montero, I. \& León, O. G. (2005). Sistema de clasificación del método en los informes de investigación en psicología. Internacional Journal of Clinical and Health Psychology, 5, 115127. Recuperado desde: goo.gl/mu8XxV

Nunally, J. C. \& Bernstein, I. H. (1994). The Assessment of Reliability. Psychometric Theory. Scientific Research. An Academic Publisher, 3, 248-292. Recuperado desde: goo.gl/NPpU34

Ospina-Rodríguez, J. (2006). La motivación motor del aprendizaje. Revista Ciencias de la Salud. Universidad del Rosario, Bogotá, Colombia, 4 (número especial), 158-160. Recuperado desde: goo.gl/uC8s2A

Oviedo, C. \& Campo-Arias, A. (2005). Aproximación al uso del coeficiente alfa de Cronbach. Revista Colombiana de Psiquiatría, 34(4), 572-580. Recuperado desde: goo.gl/vCvRVz

Palacios, A. M. \& Schinella, G. R. (2017). Diseño y validación de un instrumento para evaluar la metacognición sobre el estudio en estudiantes de medicina. Revista de la Universidad de Granada, 24, 9-28. Recuperado desde: eugra.es/index.php/reugra/article/view/70

Pérez-Gil, J. A., Chacón-Moscoso, S. \& Moreno-Rodríguez, R. (2000). Validez de constructo: el uso del análisis factorial exploratorio-confirmatorio. Psicothema, 12(2), 442-446. Recuperado desde: goo.gl/oqdDT4

Romo-Sabugal, C., Tobón, S. \& Juárez- Hernández, L. G. (2020). Diseño y validación de un instrumento para evaluar la práctica docente centrada en la metacognición en el aula. Cuadernos de Investigación Educativa, 11(2), 55-76. Doi:10.18861/cied.2020.11.2.2981

Rositas-Martínez, J. (2014). Los tamaños de las muestras en encuestas de las ciencias sociales y su repercusión en la generación del conocimiento. Innovaciones de Negocios, 11(22), 235-268. Recuperado desde: goo.gl/dTNuc9

Schraw, G. \& Dennison, R. S. (1994). Assessing metacognitive awareness. Contemporary educational psychology, 19(4), 460-475. 
Tobón, S. (2013). Formación integral y competencias. Pensamiento complejo, currículo, didáctica y evaluación. Bogotá: ECOE Ediciones.

Tamayo, O. E., Zona, J. R. \& Loaiza, Y. E. (2016). La metacognición como constituyente del pensamiento crítico en el aula de ciencias. Revista Tecné, Episteme y Didaxis: TED, número extraordinario. Memorias, Séptimo Congreso Internacional sobre Formación de Profesores de Ciencias. 12 al 14 de octubre de 2016, Bogotá. Recuperado desde: goo.gl/HGUPdw

Valle-Arias, A., Barca-Lozano, A., González-Cabanach, R. \& Núñez-Pérez, J. C. (1999). Las estrategias de aprendizaje, revisión teórica y conceptual. Revista Latinoamericana de Psicología, 31(3), 425-461. Recuperado desde: goo.gl/1MNG4D

Welch, S. \& Comer, J. (1989). Quantitative methods for public administration. Homewood, Illinois: Brooks/Cole Publishing.

Winter, J. C. \& Dodou, D. (2012). Factor recovery by principal axis factoring and maximum likelihood factor analysis as a function of factor pattern and sample size. Journal of Applied Statistics, 39(4), 695-710. 
